\title{
MODELISATION ET OPTIMISATION DE LA TREMPE DE CONTOUR DES PIÈCES DENTÉES
}

\author{
M. KCHAOU*, D. DURAND** et D. COUPARD** \\ *IPEIS: Institut Préparatoire aux Etudes d'Ingénieur de Sfax, \\ Route menzel Chaker km 0,5, BP 8053000 Sfax (Tunisie). \\ ** LAMEFIP : Esplanade des arts et Métiers, 33405 TALENCE Cedex, France
}

\begin{abstract}
RÉSUMÉ:- En général, les mécaniciens mettent en œuvre, pour assurer la fiabilité et la pérennité, des machines qu'ils conçoivent. Et pourtant, des défauts (déformation, fissures et irrégularité des profondeurs trempées) surviennent, tantôt de façon aléatoire, tantôt de façon répétée. Après avoir traité la trempe superficielle par induction des pièces de géométries simples (cylindriques) [1], nous proposons donc de traiter la trempe de contour des pièces dentées de type engrenages, roues dentées, ..., et plus précisément le profil des dents d'une roue dentées. Dans cet article nous présenterons une approche intéressante basée sur la recherche d'une fréquence optimale du générateur. Nous faisons appel à trois techniques variées: Trempe de contour mono fréquence, Trempe bi-fréquences et Trempe bi-fréquences bi-couches pour en choisir la meilleure description du contour trempé. le choix définitif de la technique sera confirmé, à la fin de ce travail, par une simulation et traitement d'un pignon.
\end{abstract}

Mots clés : Chauffage inductif, Trempe de contour, fréquence optimale, engrenage.

ABSTRACT:- In general, mechanics put in work to assure reliability and the everlastingness of machines that they conceive. And yet, of defaults (distortion, cracks and irregularity of quenched depths) occur, soon of uncertain way, soon of repeated way. After have treated the superficial quench by induction of the simple geometry pieces (cylindrical) [1], we propose to treat the quenching contour of toothed pieces type gearings, toothed wheels, therefore..., more precisely the profile of teeth of a wheel. In this paper we will present an approach interesting based on the research of an optimal frequency of the generator. We make call to three varied techniques: Quenching of contour mono frequency, Quenching bi-frequency and Quenching bi-frequency bilies to choose the best description of the contour quenched.

Key words: Inductive heating, Quenching of contour, optimal frequency, gearing

\section{INTRODUCTION}

Les traitements superficiels par induction ont pour objectif de créer en surface une mince couche de quelques dixièmes de millimètres à quelques millimètres. Cette couche, très dure et fortement précontrainte, assure une bonne résistance au sollicitations dynamiques.
L'installation de chauffage par induction, figure1, est composée de trois éléments principaux:

- La pièce à chauffer et son applicateur l'inducteur. L'applicateur est soumis à des pertes importantes et il est généralement refroidi par une circulation d'eau.

o L'adaptation, qui est un dispositif intermédiaire, permet d'adapter les caractéristiques de sortie du générateur. 


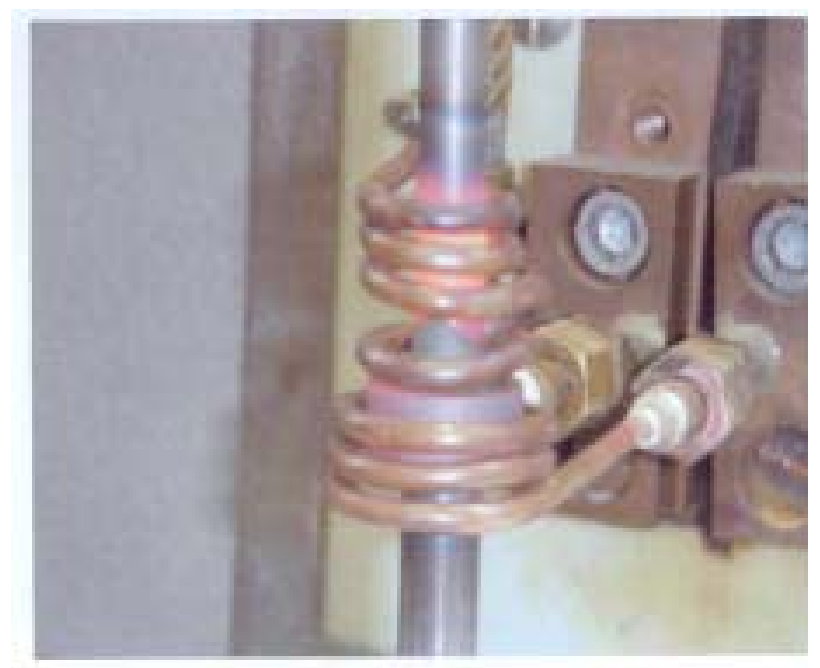

Figure 1: Procédé de chauffage superficiel par induction

o Le générateur composé de l'alimentation électrique et d'un convertisseur.

Nous avons pu montrer que le durcissement par induction remporte des résultats très encourageantes vis à vis les procédés conventionnels [1]. De même, il présente les avantages suivants:

- La breveté des cycles thermiques;

- La maîtrise des paramètres de réglages

L'aspect le plus important en chauffage par induction est de modifier les propriétés du matériau initial pour le rendre conforme aux spécifications établis par le département production. La réussite dépend de la connaissance de l'acier et comment les variations de ces constituants affecteraient les propriétés du produit final.

\section{La trempe de contour}

Dans le traitement de contour, la technique la plus utilisée est le traitement bi-fréquences par induction. Nous distinguons deux grands types de procédés en dehors des problèmes technique de réalisation qui leur sont liés.

- Le premier consiste en l'utilisation de deux sources de courant électriques: une source moyenne fréquence de 3 à $10 \mathrm{kHz}$ qui donne l'énergie nécessaire pour chauffer la massivité de la denture. Ensuite, une austénitisation, à haute fréquence de 100 à $250 \mathrm{kHz}$, du contour de la denture [2].
- Le deuxième consiste en l'utilisation de la double fréquence simultanément. Les deux sources de fréquences sont pilotés indépendamment qui sont connectées à un unique inducteur [3].

Dans le cas des engrenages et par rapport à la trempe dent par dent, la trempe de contour est le procédé le plus performant car il crée en périphérie de l'engrenage une couche durcie et précontrainte homogène en tout point [4] [5] (figure 2).

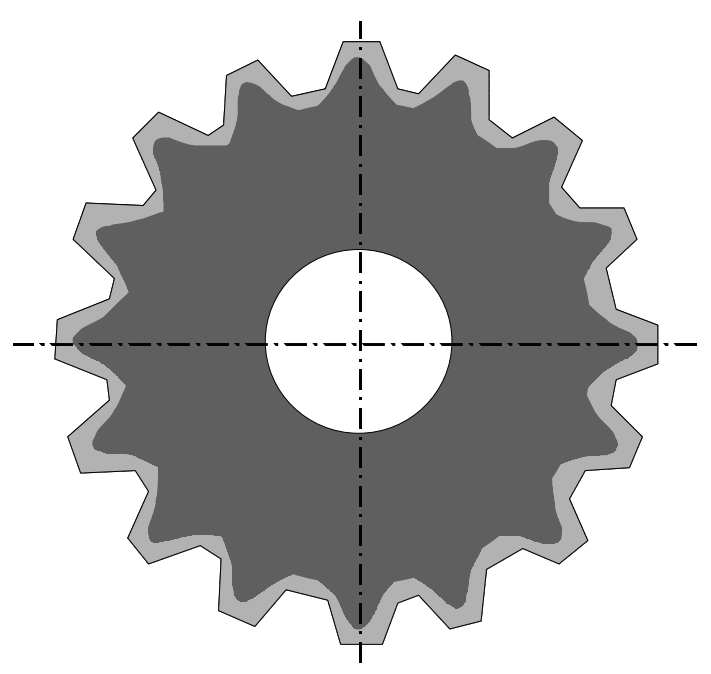

Figure 2: Trempe de contour d'engrenage

En se basant sur les travaux précédents [6], nous mis en évidence la complexité du problème auquel nous somme confronté. Nous savons que quatre grands domaines de la physique (l'électromagnétisme, la thermique, la métallurgie et la mécanique) jouent un rôle important dans le traitement superficiel utilisant le chauffage par induction.

Dans cet article nous faisons un rapide tour d'horizon de la trempe de contour utilisant l'induction. Ce traitement utilise le plus souvent une technologie bifréquences:

a) Trempe mono-fréquence

b) Trempe bi-fréquences avec transfert[7][8][9]

c) Trempe bi-fréquences simultanée[3]

d) Trempe bi-fréquences bi-couches [10][11]

\section{Effet des paramètres de réglages}

\section{Influence de la fréquence et du temps}

La bibliographie [2] [5] [10] nous indique que la fréquence et les temps de chauffage influent de façon 
forte sur la différence de profondeur chauffée entre celle mesurée au sommet et celle mesurée au pied de dent. Des moyennes fréquences et des temps importants favorisent le chauffage au pied de dent tandis que des temps faibles et de hautes fréquences favorisent le chauffage à la tête des dents. La figure 3 montre ce phénomène à travers lignes d'iso courant.

Nous constatons que les lignes d'iso courant ne sont pas parallèles à la frontière du domaine.

Si le phénomène de décroissement vers le cœur du matériau est bien visible, les effets de con-

centration dans les parties concaves (pied de dent) et leur étalement dans les parties convexes (tête de dent) induisent, pour une même valeur du champ magnétique à la surface $\mathrm{H}$, des différences notoires de densités de puissance : fortes au pied de dent, faibles au sommet pour des moyennes fréquences; faibles au pied de dent, fortes au sommet pour de hautes fréquences.(figure 4). L'explication de ces phénomènes est la suivante : Lorsque le champ $\mathrm{H}$ est constant à la frontière du domaine, les vecteurs champs électriques $\mathrm{E}$ décroissent dans la pièce de façon identique de la surface vers le cœur quelle que soit la courbure de la frontière. Les courants eux se répartissent selon la loi de Kirchhoff [10], de façon inversement proportionnelle à la résistance des chemins possibles.

Bien que la circulation des vecteurs intensité soit solénoïdale, la puissance dissipée dans une section normale n'est pas constante quelle que soit la section car

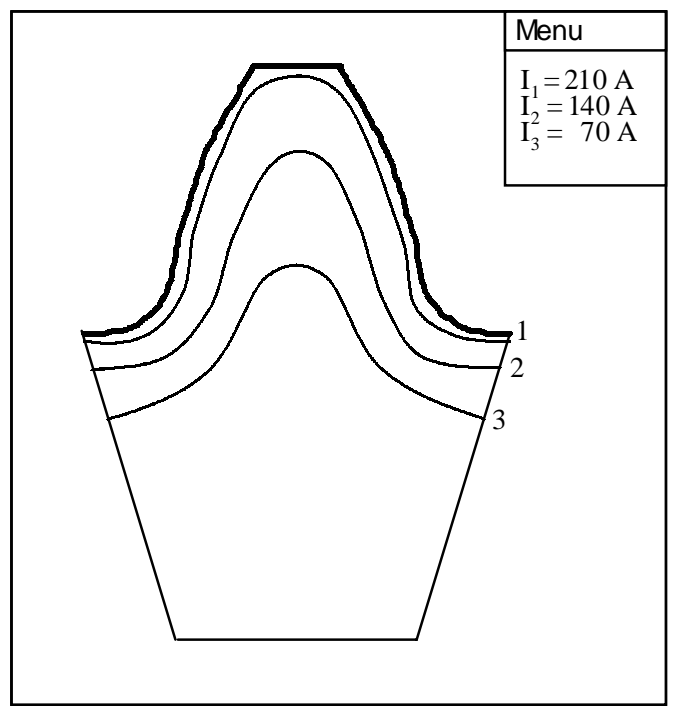

a) moyenne fréquence elle dépend via le facteur $\mathrm{i}^{2}$ de la répartition des courants. Plus ils seront concentrés en surface (région concave) et plus la puissance dégagée dans cette section sera importante.

\section{Cinétique thermique}

Par rapport à la profondeur chauffée les dents présentent une très faible massivité tandis que le corps de l'engrenage en a une très forte.

Pour réaliser une trempe de contour de la dent il faut employer une fréquence élevée et des temps très brefs. En pieds de dents bien que l'énergie dissipée soit plus importante la conduction thermique réduit considérablement la profondeur chauffée (figure $5 \mathrm{~b}$ ). Si l'on veut chauffer le pied de dent il faut des fréquences basses et des temps importants (figure 5a).

Dans le même ordre d'idée, nous savons que l'arrêt de la source ne provoque pas forcément l'arrêt de la montée en température de tous les points. La cas de la denture est très représentatif de ce point de vue, car nous constatons la progression de l'isotherme Ac1 (figure6) vers le cœur de la dent, alors que partout ailleurs il recule. De même, l'obtention d'une trempe de contour est incompatible avec retard douche.

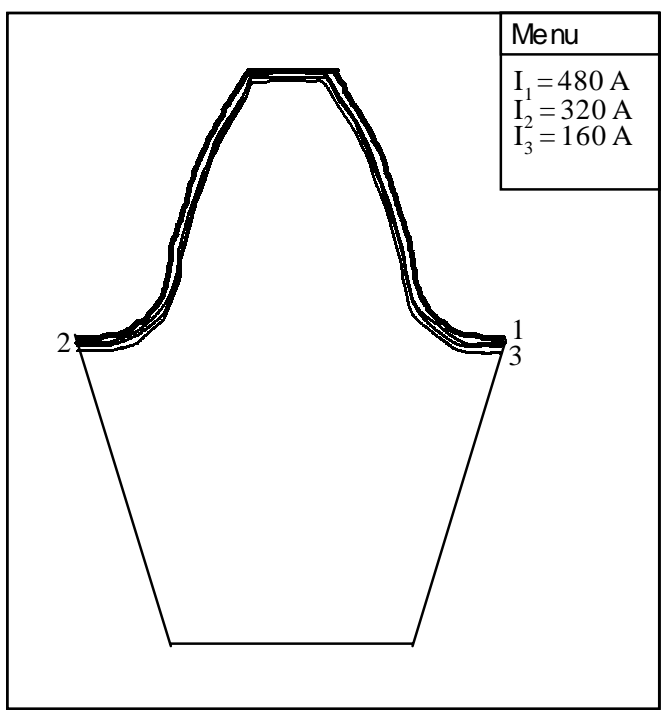

b) haute fréquence

Figure 3: Lignes d'iso courant 


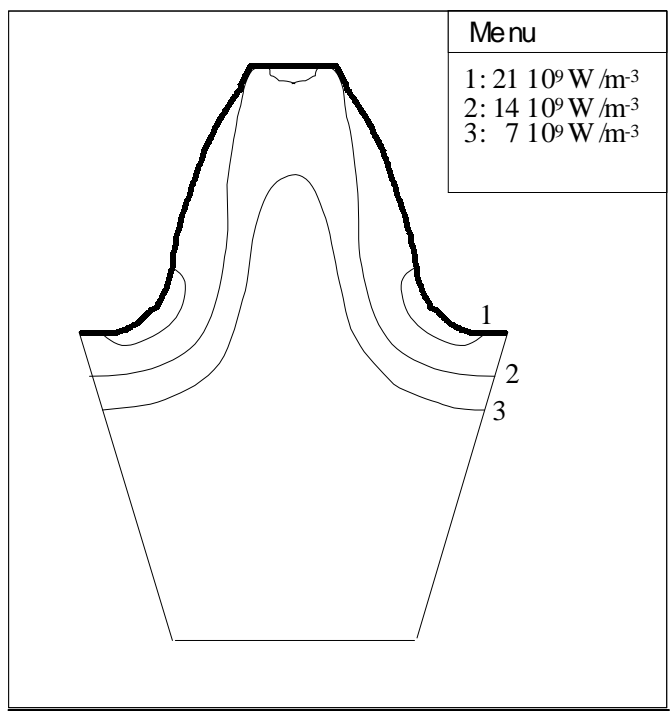

a) moyenne fréquence

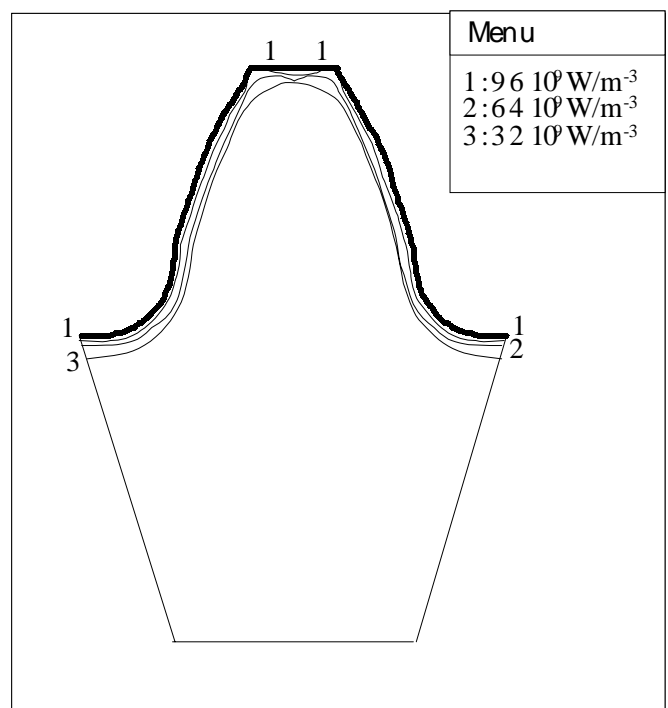

b) haute fréquence

Figure 4: lignes d'iso densité de puissance

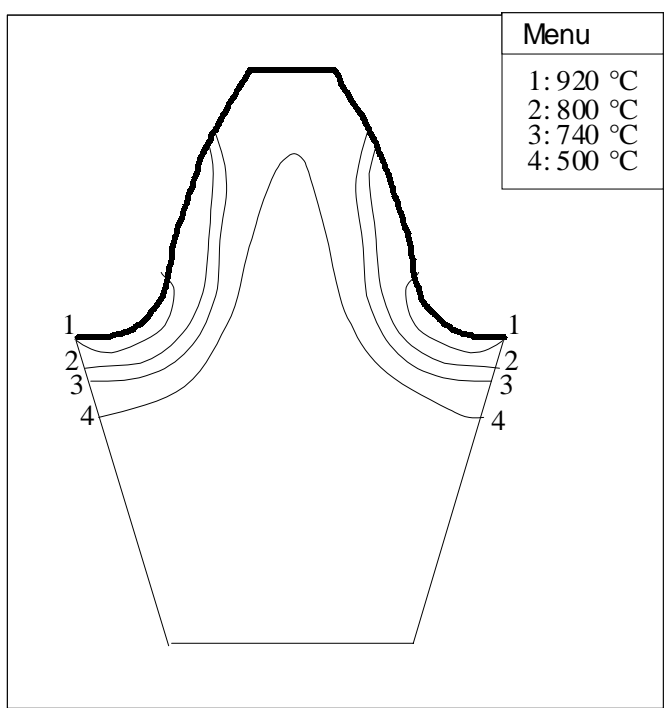

a) moyenne fréquence

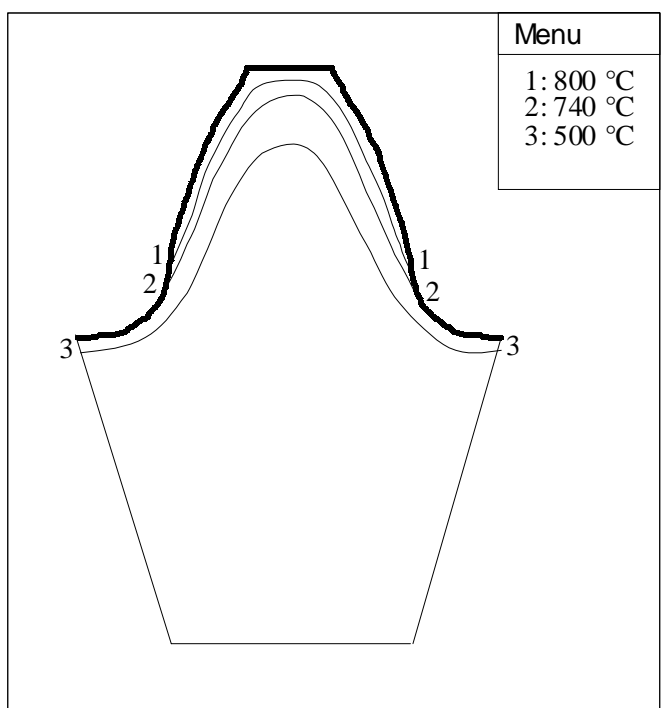

b) haute fréquence

Figure 5: lignes isothermes 


\section{Trempe de contour mono fréquence}

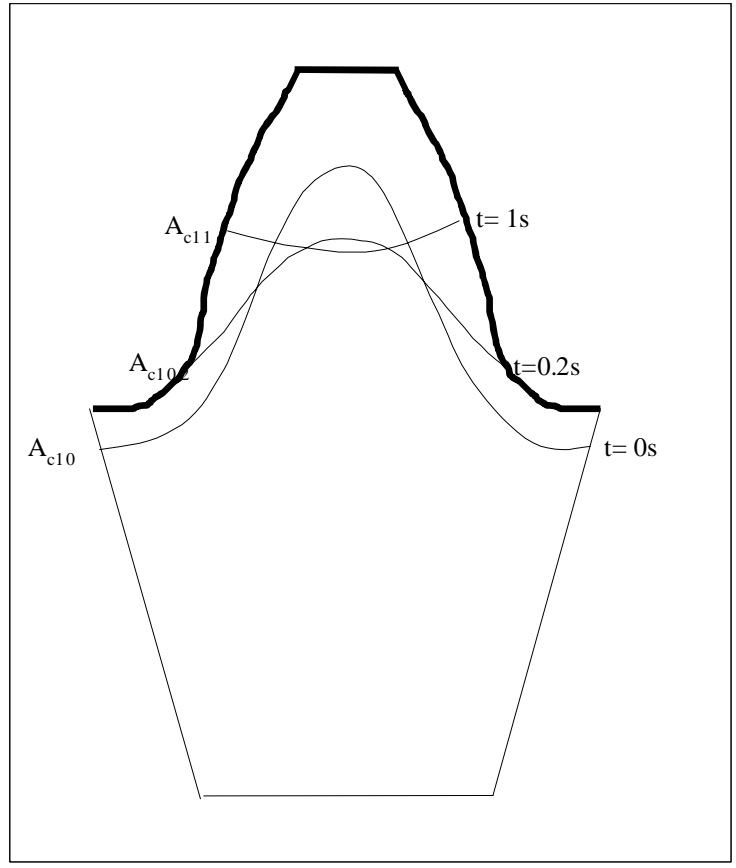

Figure 6: influence du retard douche
Pour chaque type d'engrenage, il existe un triplé fréquence temps, densité de puissance qui assure une austénitisation de contour. Cette méthode présente trois inconvénients:

Le triplé des paramètres de réglage, est très délicat à déterminer expérimentalement. La détermination par simulation est lente. De plus il faut travailler à une fréquence bien déterminée, lorsque le diamètre primitif où le module change, il faut changer de fréquence.

Les variations de dureté et les variations des contraintes résiduelles qui y sont associées génèrent une résistance à l'usure dégradée. Il faut donc effectuer le traitement à partir d'une structure plus homogène à savoir une structure trempée et revenue.

Outre les temps importants nécessaires à ces traitements et la dégradation de l'usinabilité qu'en résulte, la trempe à cœur nécessite des aciers contenant des éléments d'addition qui les rendent plus coûteux.

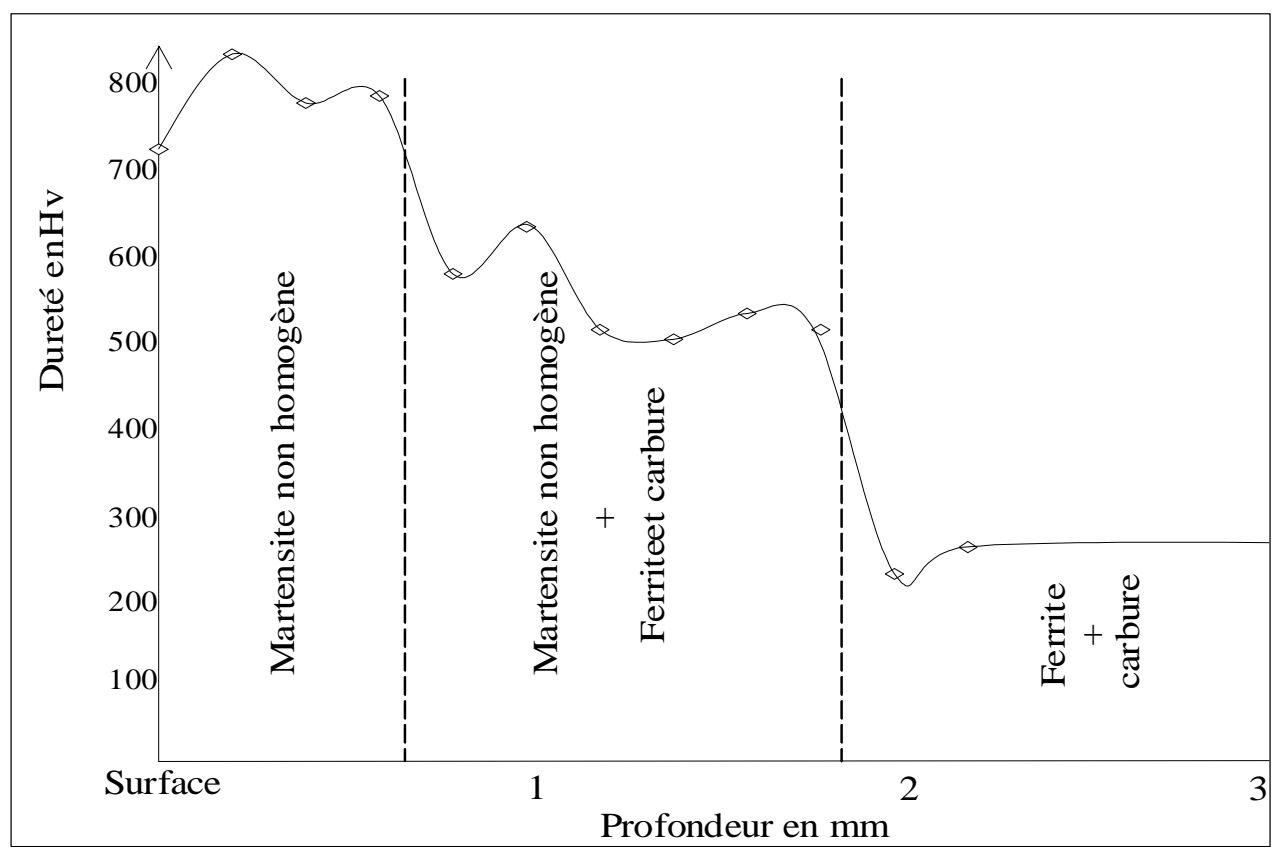

Figure 7 : filiation de dureté dans la zone trempée pour une structure férrito-perlitique 


\section{Trempe bi-fréquences}

La bi-fréquences est le procédé le plus rapide connue pour le chauffage des dentures. La trempe bi-fréquences consiste à effectuer un chauffage qui chauffe prioritairement les pieds de dents et l'intervalle entre deux dents, à moyenne fréquence (6 à $12 \mathrm{k} \mathrm{Hz}$ ) et un deuxième chauffage à haute fréquence (100 à 200 k Hz) qui privilégie le pourtour de la dent et assure ainsi un chauffage de contour.
L'intérêt de ce type de traitement est la possibilité d'employer des fréquences standards et de multiplier les plages de réglage possibles.

Trois variantes de ce traitement sont employées:

\section{Deux inducteurs et transfert de la pièce}

La figure 8 représente le cycle utilisé pour le chauffage en mode bi-fréquence.

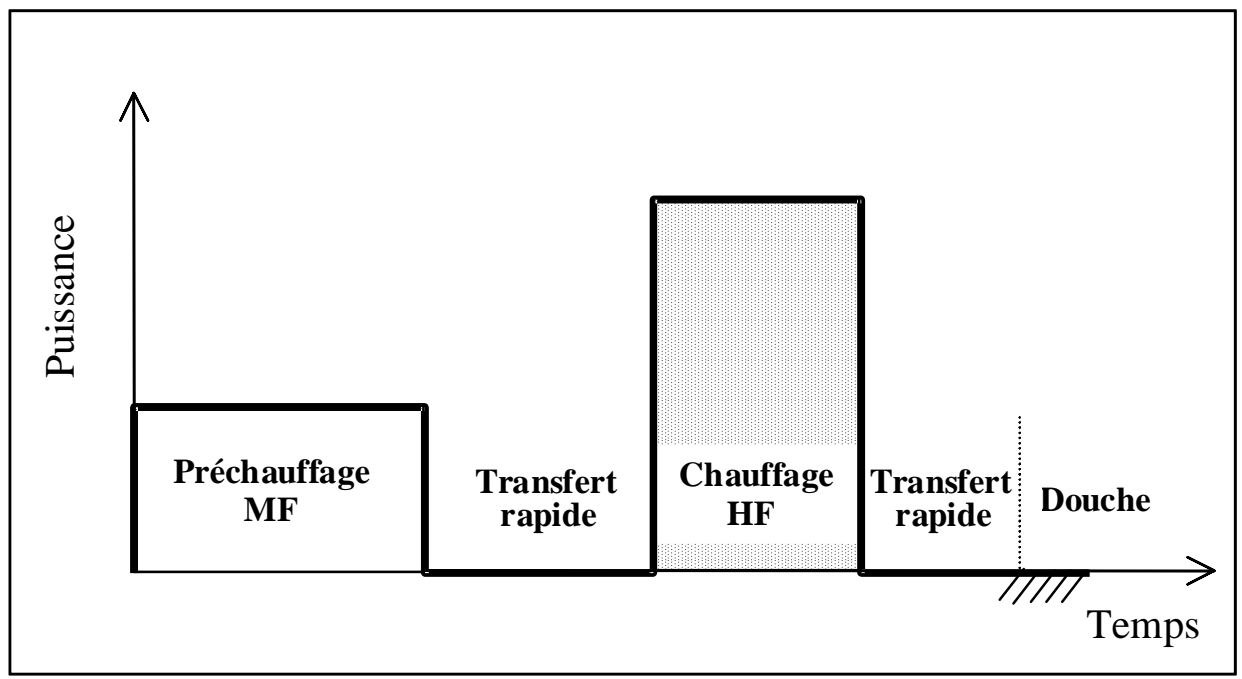

Figure 8: cycle de traitement

Ce traitement peut s'effectuer avec des générateurs à fréquences standards. Cependant les temps de transfert doivent être très courts (quelques dixième de seconde) sinon la température de préchauffage baisse considérablement et la température dans la dent s'égalise.

Comme dans le cas précédent ce traitement doit s'effectuer sur des aciers trempés revenus

\section{Chauffage simultané bi-fréquences}

Cette deuxième méthode consiste à utiliser deux sources de puissance pilotées indépendamment et travaillant à deux fréquences différentes pour alimenter simultanément un même inducteur. Ce mode peut présenter des difficultés techniques, réglages des temps d'utilisation des sources, changement de régime de l'inducteur,...
Les avantages de cette méthode sont l'utilisation de temps de cycle court. Les fréquences travaillant en même temps et pas de façon consécutive, et offre un large degré de liberté pour ajuster le profil du traitement en fonction de la taille et du type de denture.

\section{la trempe bi-fréquences bi-couches.}

Elle s'applique sur des couronnes recuites (structure ferito- perlitique). Deux fréquences sont employées l'une de 5 à $10 \mathrm{kHz}$ dite moyenne fréquence et l'autre de 100 à $200 \mathrm{kHz}$ dite haute fréquence. Le traitement comporte quatre séquences décrites à la figure 9: Préchauffage des pieds de dents (figure 10), Trempe de contour profond de la dent(figure 11), Préchauffage et revenu de la $1^{\text {er }}$ couche trempée (figure 12), Trempe de contour mince (figure 13). 


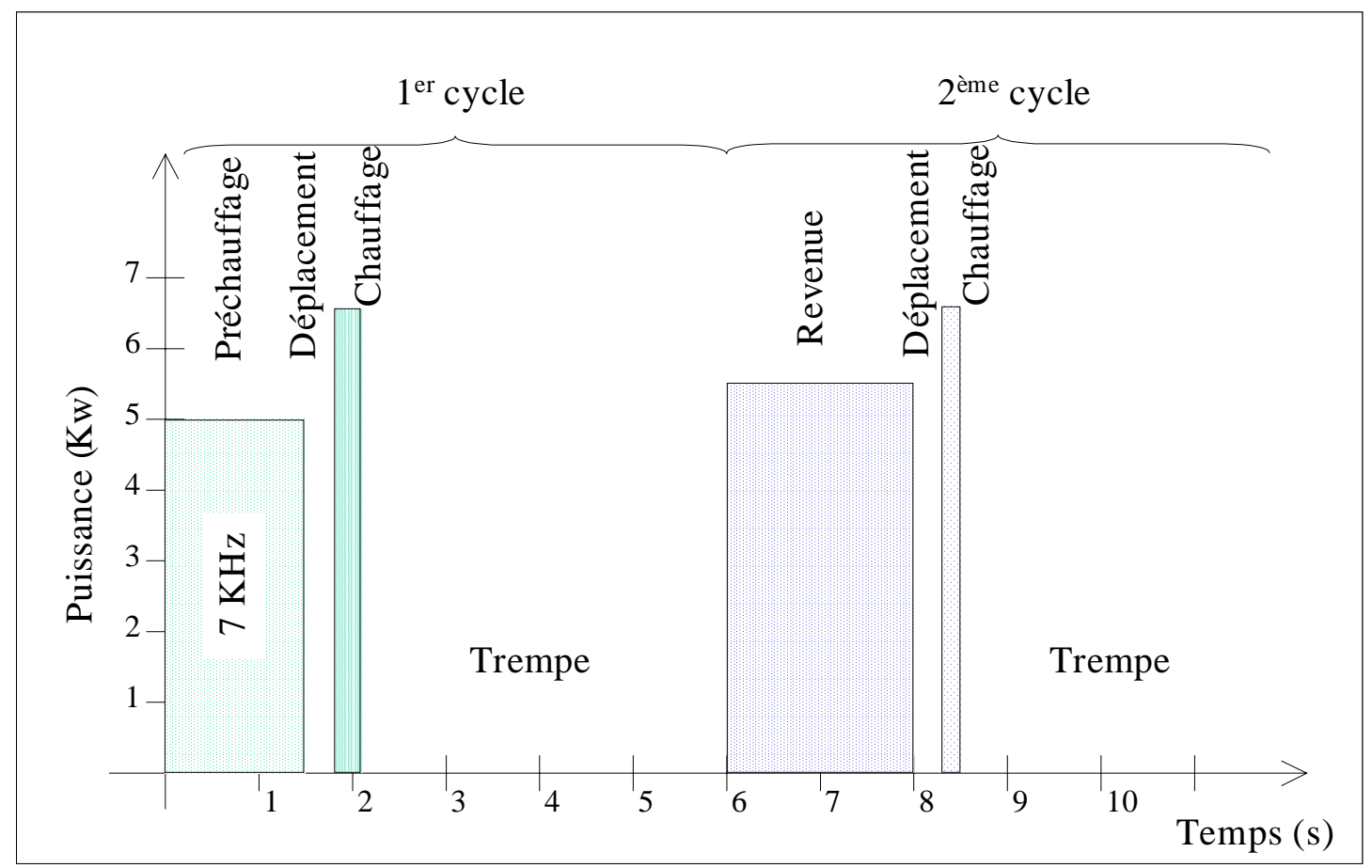

Figure 9: cycle de traitement simulé sur une denture

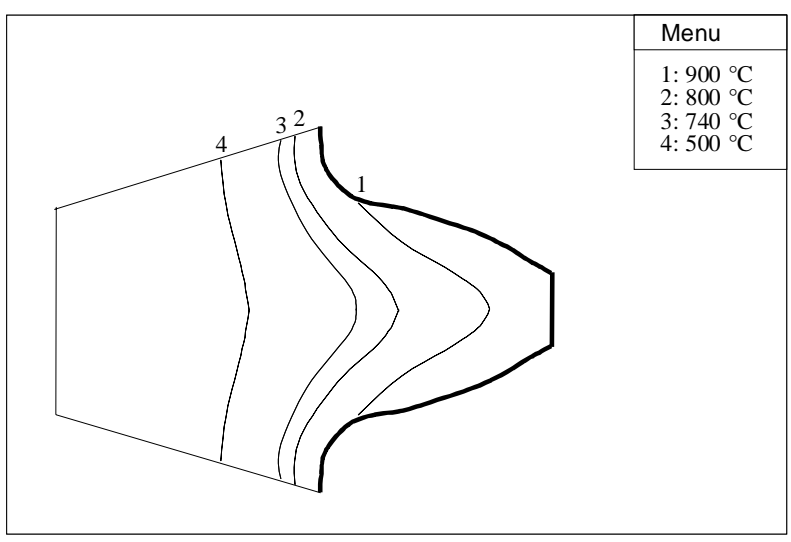

Figure 10 : Préchauffage des pieds de dents

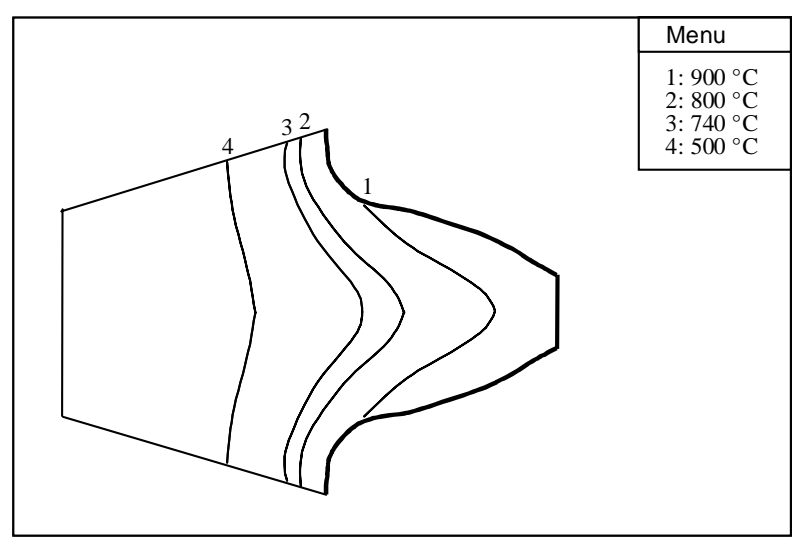

Figure 11 : Trempe de contour profond de la dent 


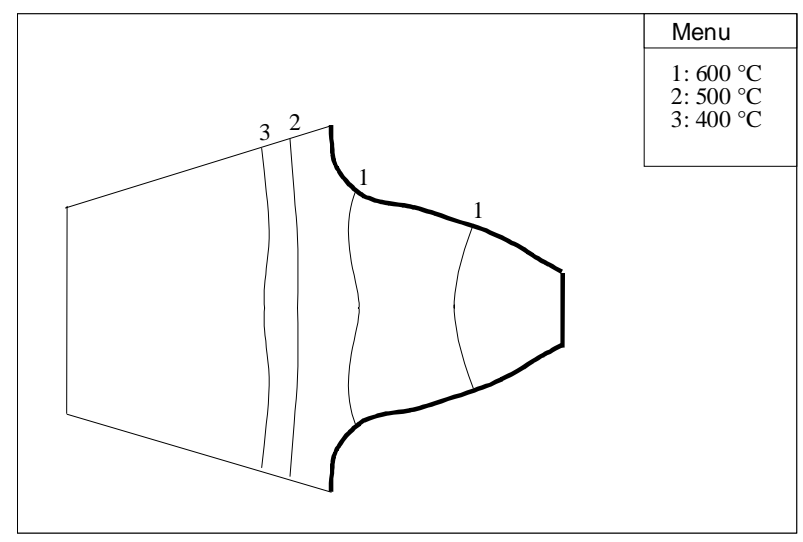

Figure 12: Préchauffage et revenu de la ${ }^{\mathrm{er}}$ couche trempée

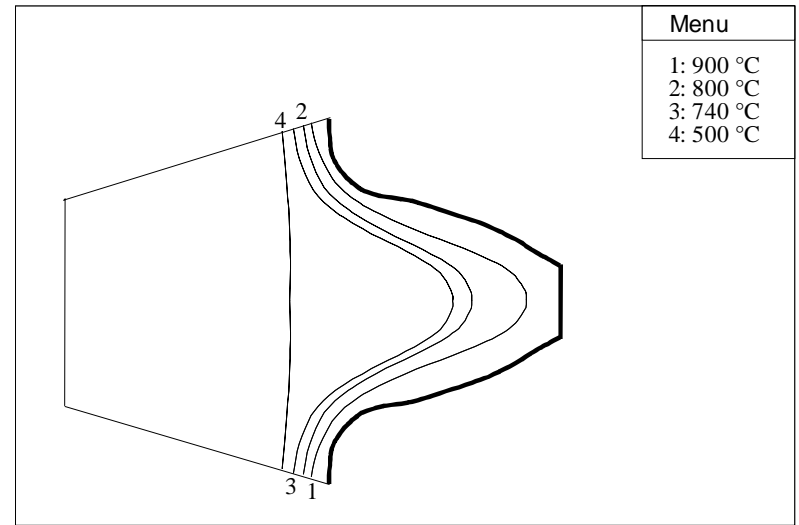

Figure 13: Trempe de contour mince
Comme nous l'avons vu pour le cylindre [1], le chauffage se décompose en différentes étapes. Les figures 10, 11, 12 et 13 montrent un exemple de cycle de chauffage susceptible de donner un profil de trempe respectant le contour de la denture.

\section{Résultat expérimental}

Soulignons qu'un certain nombre de paramètres s'écartent de ceux choisis pour le cylindre, notamment la fréquence et les cinétiques thermiques, du fait de la différence de géométrie entre les deux pièces. La géométrie de l'applicateur (inducteur) est ici en cause ainsi que le support. Les niveaux de dureté de surface sont de l'ordre de $750 \mathrm{Hv}$ que se soit en sommet, flanc et pied de denture. La profondeur de trempe au sommet est plus important qu'à celle au pied (de l'ordre $0,5 \mathrm{~mm}$ ). Malgré cette irrégularité, il reste en concordance avec les résultats de simulation.

Le figure 14 montre les dents après la première trempe de contour et la deuxième trempe. Deux difficultés technologiques sont à surmonter pour maîtriser un tel traitement.

1. Les temps de transfert de la moyenne à la haute fréquence doivent être très brefs (fraction de seconde) si l'on veut que la préchauffe du pied de dent reste significative;

2. Les temps de monté en puissance du générateur HF doivent être extrêmement brefs (quelques dizaine de secondes).

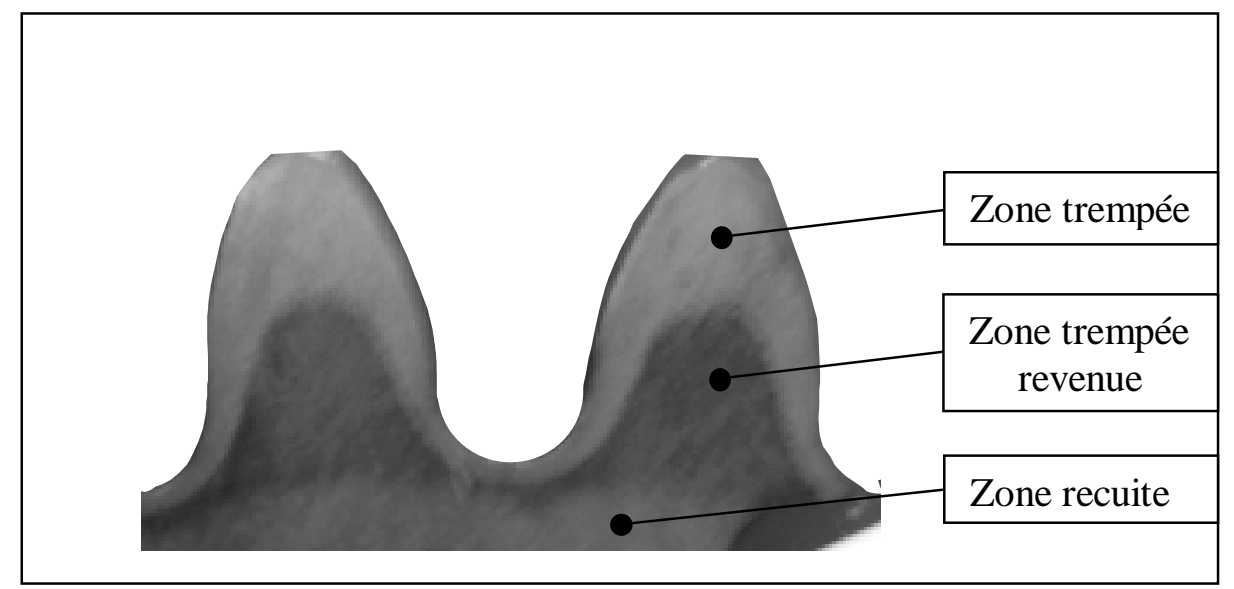

Figure 14 : Pièce trempée revenue 


\section{CONCLUSION}

Les résultats obtenus sur les cylindres [1] autant que sur les dentures semblent encourageantes.

Toutefois, si cette partie constitue pour la simulation des phénomènes métallurgiques et mécaniques, il est nécessaire de le consolider par une caractérisation du matériau plus complète et une recherche des paramètres d'influence ainsi qu'une compagne d'essais.

Nous avons montré qu'il est possible de traiter des pignons de faible module $(3 \mathrm{~mm})$ en trempe de contour. Pour cela nous avons utilisé la double fréquence et la double couche. En cumulant ces deux spécificités du traitement, nous parvenons à un traitement tout à fait en accord avec les exigences imposées par l'industriel.

Le traitement remplit en effet sa fonction de trempe superficielle avec des niveaux de dureté et de profil de trempe adéquats.

Pour arriver à ce résultats, notre démarche de mise au point des paramètres procédés de traitement et nos essais sur éprouvette a montré son intérêt. Elle nous a permis d'une part de mettre au point le cycle de traitement et d'autre part de dimensionner l'installation comprenant le générateur et la géométrie des pièces.

Quelque soit la méthode employée la trempe de contour nécessite:

- le réglage pointu de plusieurs facteurs (puissances, fréquences)

- un respect des temps très serré.

Dans ce cadre, la simulation numérique est une aide très précieuse qui permet de réduire considérablement le nombre d'essai et qui surtout permet de prédéterminer le matériel nécessaire: type de générateur (fréquence, puissance, temps de montée en charge) et la performance du dispositif de transfert.

La trempe bi-couche, quant à elle, semble promise à un avenir certain et pas seulement pour la pignonerie. Elle permet,sur des pièces massives, en acier peu ou pas alliée à structure férito-perlitique, d'obtenir des performances d'endurance comparables à celle faîte d'acier plus allié.

\section{REFERENCES}

[1] M. Kchaou, D. Durand, J.P. Sagaspe (1996). Management of materials properties in an electrical thermal metallurgical simulation of quenching following inductive surface. Journal of materials Engineering and Performances, Vol. 5 n 2 , p.192200

[2] J.M. Storm and M. R. Chaplin (1987). Induction gear hardening by dual frequency method. Journal of Heat treating vol. $19 \mathrm{~N}^{\circ}$,p. 30-37.

[3] L. Markegard (1991). Two-frequency system for surface hardening of gears. Proceeding of the European congress Induction and Industrial applications Strasbourg, p. 12.

[4] C.P. Hunter, H. Michaud, and G. Baudry (1996). Fatigue behaviour of gear steels rohich have been subjected to surface treatment Internationaux de France du traitement thermique Reims, ATTT 96, p.105-116.

[5] B. Criqui, F. Lestrat, M. Frainais, F. Ruckstuhl, C hunter, et C. Picard (1996). Tenue en fatigue de pièces de pignonnerie traitées par trempe de contour.b Internationaux de France du traitement thermique Reims, ATTT 96, p. 117-126.

[6] M. Kchaou (1995). Contribution à la prédétermination des états structuraux et des contraintes residuelles après trempe superficielle par induction. Thèse de doctorat ENSAM Bordeaux, numéro d'ordre 95.03, p. 147.

[7] O. Longeot D. Durand and P. Wendling (1990). Induction: use of dual frequency sources. The fourth biennial IEEE conference in electromagnetic field computation. Toronto, oct.22-24 90, proceedings, p.134-136.

[8] G.A.Oakley (1990). Contour Hardening of gears by the Dual-pulse induction method. Heat treatment of metals, vol. $n^{\circ} 4$, p. 99-101.

[9] J.P. Horvath (1995). Process development for dual frequency induction hardening at Allison transmission. Proceedings of the $1^{\text {st }}$ international conference «induction hardened gears and component Indianapolis, CMF, p. 3-7. 
[10]C. Delaleau (1997). Simulation 2 D, 3D du procédé de traitement thermique utilisant le chauffage par induction à source bi-fréquence Application au durcissement superficiel bi-couche de pignons de boîte de vitesse. Thèse de doctorat ENSAM Bordeaux $\mathrm{n}^{\circ} 9730$, p. 165.

[11] B. Criqui, S. Plano, F. Ruckstuhl, B. Oltosson, O. Longeot et C. Delaleau (1997). Multi-fréquency induction hardening of gears to replace carburizing. Methodology of development. The $17^{\text {th }}$ ASM heat treatment conference and exposition $-1^{\text {st }}$ international induction heat treating symposium. Indianapolis..
[12] O. Longeot, D. Durand and J.L (1988). Lebrun. Characterisation test for induction heat treatment. Application to C 42 and 42 Cr 2 steels. Proceedings of the $6^{\text {Th }}$ international congress on heat treatment of materials Chicago, (Ed ) ASM international, p. $143-150$

[13]R. Ernst , P. Grosse, L. Philippe (1999). An electromagnetic-thermal coupling example solved solved on a high frequency inductive system with the matlab finite element solver. International journal of thermal sciences, vol. 38, p. 481-491. 